\title{
Utilization of Graphene Liquid Cells Electron Microscopy (GLC-TEM) to Study In- situ ferritin Biomineralization
}

\author{
Surya Narayanan, Reza Shahbazian-Yassar and Tolou Shokuhfar \\ University of Illinois at Chicago, Chicago, Illinois, United States
}

Neurodegeneration by brain iron accumulation (NBIA) is a heterogeneous form of neurological disorder wherein iron accumulation is witnessed in the basal ganglia of the brain. Studies have shown that the iron accumulation can cause progressive degeneration of nerve cells, resulting in neurological diseases such as Alzheimer's disease (AD), Parkinsonism, retinal degeneration, and neuropsychiatric disorder [1]. While the mechanism of disease progression or the cure has still not been discovered, one aspect that is still questioned is the body's own physiological mechanism of iron regulation. In a healthy human, excess of iron in the cytoplasm is regulated by iron storage proteins called as ferritins. Through series of chemical reactions (Figure 1), ferritins convert the free radical $\mathrm{Fe}^{2+}$ iron ions to a stable $\mathrm{Fe}^{3+}$ iron oxide core, thus facilitating storage. In events of iron deficit, the stored iron oxide core dissolves to release $\mathrm{Fe}^{2+}$ iron ions back into the system. Despite this fact, iron accumulation in the brain and its adverse effect is pronounced. Studying the structure and chemistry of the iron core can unfold the biomineralization pathways while serving as a potential biomarker for neurological diseases.

Traditionally, transmission electron microscopy (TEM) has been used as one of the techniques to study the iron oxide core of ferritin [2]. Through decades of research, the size, morphology, structure, and the chemical composition of the core have been identified. However, the intricacy in the outer protein structure and the miniaturized size of the inner core has made it challenging to observe these mechanisms in real-time. While there have been considerable efforts to study the biomineralization in ferritin using conventional TEM, cryogenic approaches as well as $\mathrm{Si}_{3} \mathrm{~N}_{4}$ based fluid cells, the major setback with these techniques is the ability to obtain both spatial as well as temporal resolution. Consequently, the conclusions of research were based on the average results obtained from several proteins across the area of the grid. While these results facilitate an understanding of generic mechanism, the possibility of differences in each protein cannot be neglected. In this study, we utilized graphene liquid cells (GLCTEM) to obtain information from a single ferritin without compromising the quality and accuracy of the results. Due to its ultrathin membrane and the ability to mitigate radiation damage, GLC-TEM has been one of the viable options to study biomineralization in ferritin in real-time.

Biomineralization in apoferritin (ferritin without iron) was triggered through synthetic means via the wellestablished ex-situ biochemical reaction. The concentration ratios of iron/apoferritin in the solution were fixed based on the results obtained through the UV-visible spectroscopy. Further, the solution mixture was encapsulated in GLC-TEM to observe the in-situ biomineralization in real-time. The formation of iron oxide core was monitored through the changes in the intensity of the high angle annular dark field (HAADF-STEM) image (Figure 2). This was further validated through the chemical changes in the core measured via the electron energy loss spectroscopy (EELS) which indicated the changes in the nitrogen $(\mathrm{N})$, oxygen $(\mathrm{O})$, and iron $(\mathrm{Fe})$ signal during biomineralization. During the zeroth hour, we observed $\mathrm{N}$ and O signal from the protein, however the Fe signal was absent. However, after an hour of biomineralization, a dominant Fe peak was observed from the same protein. The iron peaks formed during this process was further analyzed to understand the chemical composition of the core at each stages of biomineralization, It was observed that the core primarily forms iron oxides composed of both $\mathrm{Fe}^{2+}$ and $\mathrm{Fe}^{3+}$ ions which 
gradually convert to $\mathrm{Fe}^{3+}$ form. The results obtained from this study are promising to develop potential biomarkers for many neurological conditions [3].

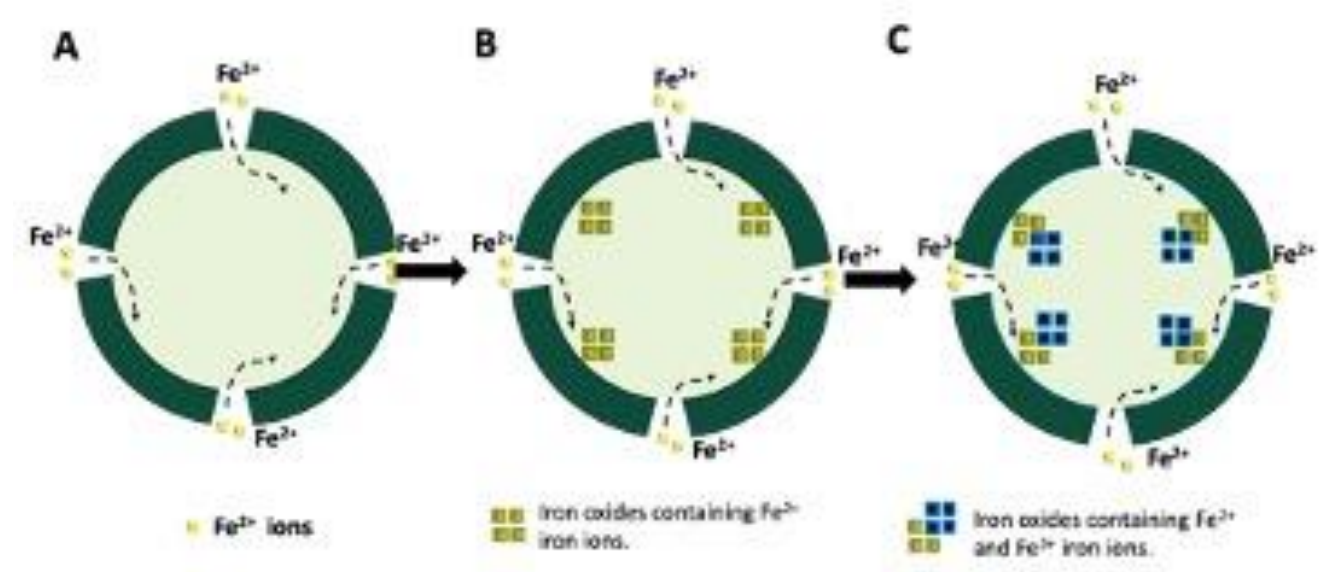

Figure 1. A schematic showing the biomineralization process in ferritin. A. The soluble iron $\left(\mathrm{Fe}^{2+}\right)$ enters the protein. The iron ions move towards ferroxidase sites for iron oxidation and nucleation. B. The iron nucleation results in the formation of islands of iron oxides nuclei with the protein. $\mathbf{C}$. The nuclei further grows with the incoming iron ions to form the fully grown iron oxide core. The final iron oxide core is considered to be similar to ferrihydrite mineral core.
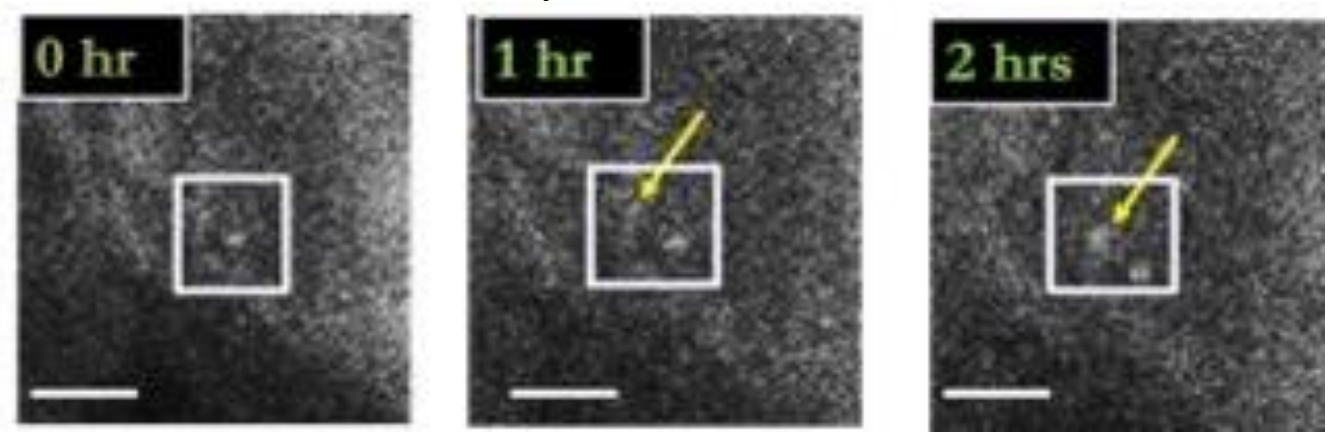

Figure 2. The different stages of in situ biomineralization in ferritin observed via the GLC-TEM technique.At the onset of biomineralization at zeroth hour, there is no iron oxide formation inside the proteins. As a result, the intensity of the HAADF STEM image is very low and the protein is barely seen. This can be observed in the region of interest (white color box) during the zero hour, wherein only a single protein is observed. However, the intensity of the HAADF STEM image changes with time. As a result, the iron oxide core of the second protein (in the region of interest)is observed indicated by yellow color arrow (after $1 \mathrm{hr}$ and $2 \mathrm{hrs}$ ). The scale bar of the images are $100 \mathrm{~nm}$.

\section{References}

[1] A.Gregory, BJ Polster, and SJ Hayflick, Journal of medical genetics, 46(2), (2009),pp 73-80.

[2] EC. Theil, Inorganic Chemistry, 52(2013), 21

[3] The authors acknowledge funding from the National Science Foundation- CAREER award- Grant NoDMR- 1564950. Dr Tolou Shokuhfar is acknowledged for her many useful discussions and contribution to this work. This work made use of instruments in the Electron Microscopy Service (Research Resources Center, UIC) 